\title{
Meliaceae (Sapindales): riqueza y distribución en el estado de Tabasco, México
}

\section{Meliaceae (Sapindales): species richness and distribution in Tabasco, Mexico}

\section{Acta Botanica Mexicana}

\author{
Doris Córdova-Córdoval (iD), Carlos Manuel Burelo-Ramos,4 (iD), Manuel J. Campos-Díaz' (iD, Neil Ebeth Meled Morales- \\ Rodríguez' (D), William Álvarez-Jiménez’ (D), Graciela Beauregard Solís² (D), Nahúm Muñiz-Chavarríal (iD, Marynor Elena \\ Ortega-Ramírez ${ }^{3}$
}

\section{Resumen:}

Antecedentes y Objetivos: Con la finalidad de actualizar el listado de especies de Meliaceae en el estado de Tabasco bajo el sistema de clasificación actual, se completó una revisión taxonómica de la familia, como contribución al proyecto "Flora de Tabasco".

Métodos: Se llevó a cabo una revisión en herbarios estatales y nacionales de los ejemplares de las especies de la familia Meliaceae y se realizaron colectas en campo de 2015 a 2017 en vegetación secundaria y primaria del estado. El material colectado fue identificado por medio de claves taxonómicas y cotejado con material de herbario. También se consultaron los ejemplares tipo disponibles en JSTOR Global Plants. Se hizo una clave de identificación para las especies de la familia en Tabasco y un análisis de distribución por tipo de vegetación y datos ecológicos; así mismo se registran nombres comunes y se ilustran algunas de las especies.

Resultados clave: La familia Meliaceae está representada por 11 especies en cuatro géneros nativos y dos especies en dos géneros introducidos. Se registran por primera vez dos especies para el estado. El género Trichilia es representado por siete especies, seguido de Guarea con dos. Los municipios con el mayor número de especies son Centro y Teapa con ocho especies cada uno y Balancán con siete.

Conclusiones: Las Meliáceas nativas presentes en la zona de estudio representan $45.83 \%$ de las 24 especies nativas estimadas para la familia en México y están distribuidas principalmente en áreas de vegetación secundaria y manchones de selva en municipios serranos fronterizos con Chiapas y Guatemala. Palabras clave: biodiversidad, clave dicotómica, especies maderables, taxonomía.

\section{Abstract:}

Background and Aims: In order to update the checklist of Meliaceae that occur in the state of Tabasco under the current classification system, a taxonomic review of the family was conducted, contributing to the "Flora de Tabasco" project.

Methods: A revision of specimens belonging to Meliaceae was performed in both state and national herbaria. Field work was carried out between 2015 and 2017 sampling a wide array of of natural and secondary vegetation types in Tabasco. The collected material was identified with the use of taxonomic keys and compared with reliably identified herbarium material. Type specimens available in JSTOR Global Plants were also consulted. We provide an identification key for the species of the family in Tabasco. We also performed an analysis of the distribution of the species based on vegetation type and ecological data. Finally, we provide a list of common names and images of several species.

Key results: The Meliaceae are represented by 11 species and four native genera; there are also two alien genera (each represented by a single species). Two species constitute new records for the flora of Tabasco. Trichilia is represented by seven species, whereas Guarea has two. The municipalities with the highest number of species are Centro and Teapa with eight species each and Balancán with seven species.

Conclusions: The Meliaceae species reported for the study area represent $45.83 \%$ of the 24 native species of this family known in Mexico. They are mostly distributed in areas of secondary vegetation and remnants of tropical rain forest close to the borders with Chiapas and Guatemala.

Key words: biodiversity, dichotomous key, taxonomy, timber species.

${ }^{1}$ Universidad Juárez Autónoma de Tabasco, División Académica de Ciencias Biológicas, Herbario UJAT, Carretera Villahermosa-Cárdenas km 5 s.n., entronque a Bosques de Saloya, 86150 Villahermosa, Tabasco, México.

Universidad Juárez Autónoma de Tabasco, División Académica de Ciencias Biológicas, Carretera Villahermosa-Cárdenas km 5 s.n., entronque a Bosques de Saloya, 86150 Villahermosa, Tabasco, México.

${ }^{3}$ Universidad Autónoma de Chiapas, Facultad Maya de Estudios Agropecuarios, Carretera Catazajá-Palenque km 4, 29980 Catazajá, Chiapas, México.

${ }^{4}$ Autor para la correspondencia: carlos.burelo@ujat.mx
Recibido: 3 de junio de 2020.

Revisado: 12 de junio de 2020.

Aceptado por Marie-Stéphanie Samain: 15 de julio de 2020.

Publicado Primero en línea: 17 de agosto de 2020.

Publicado Acta Botanica Mexicana 127 (2020).
Citar como: Córdova-Córdova, D., C. M. Burelo-Ramos, M. J. Campos-Díaz, N. E. M. Morales-Rodríguez, W. Álvarez-Jiménez, G. Beauregard Solís, N. Muñiz-Chavarría y M. E. Ortega-Ramírez. 2020. Meliaceae (Sapindales): riqueza y distribución en el estado de Tabasco, México. Acta Botanica Mexicana 127: e1741. DOI: 10.21829/abm127.2020.1741 


\section{Introducción}

Se estima que a nivel mundial existen ca. 500 especies con valor comercial en el mercado internacional de la madera (López-Camacho y Cárdenas-López, 2002). La familia Meliaceae contiene varias especies maderables altamente valoradas y cotizadas en el mercado internacional (principalmente de los géneros Cedrela P. Browne, Swietenia Jacq., Khaya A. Juss. y Entandrophragma C. DC.) y son consideradas de las más atractivas en el mundo por el lustre, color, grano y la resistencia al ataque de insectos (Pennington et. al., 1981). Lo anterior ha traído una serie de problemas asociados al valor económico que estas especies presentan, pues han sido sometidas a la tala ilícita y al transporte ilegal para un mercado de madera cada vez más exigente, provocando problemas de sobreexplotación, pérdida del hábitat, expansión de los asentamientos humanos y posiblemente erosión genética (Rodan et al., 1992; Veríssimo et al., 1998; Laurance, 1999; Gullison et al., 2000; O'Neill et al., 2001; Kometter et al., 2004; Cornelius et al., 2005; Thiago et al., 2008). Se tienen registradas 358 especies de Meliaceae incluidas en la Lista Roja de la IUCN (International Union for Conservation of Nature) (IUCN, 2020). Así mismo, tres especies de Swietenia se enumeran en el apéndice II del CITES (Convention on International Trade in Endangered Species of Wild Fauna and Flora) (CITES, 2020): Swietenia humilis Zucc., S. macrophylla King y S. mahagoni (L.) Jacq., mientras que en el apéndice III se mencionan poblaciones de Cedrela fissilis Vell., C. lilloi C. DC. y C. odorata L.

La familia Meliaceae está ubicada en el orden Sapindales junto con Anacardiaceae, Biebersteiniaceae, Burseraceae, Kirkiaceae, Nitrariaceae, Rutaceae, Sapindaceae y Simaroubaceae (APG IV, 2016), presenta una distribución pantropical e incluye generalmente árboles que habitan desde los bosques tropicales y manglares hasta los semidesiertos (Muellner et al., 2003). Meliaceae exhibe un amplio rango de caracteres morfológicos principalmente en sus inflorescencias, frutos y semillas, lo que ha provocado una historia taxonómica confusa, siendo la monografía de Pennington y Styles (1975) la que contribuyó a esclarecer su circunscripción genérica; así mismo mejoró la clasificación de tribus y subfamilias. La historia taxonómica complicada tiene su origen debido a la naturaleza difusa y a menudo reticulada de la variación que parece haber sido producto de varios paralelismos evolutivos y de forma independiente en inflorescencias, frutos y semillas. Debido a esto, algunos géneros y la mayoría de las tribus solo pueden ser diagnosticados mediante una combinación de varios caracteres, por lo que encontrar un carácter como una herramienta de diagnóstico puede ocasionalmente ser poco confiable (Muellner et al., 2003).

Se reconocen 48-53 géneros y 700-702 especies de Meliaceae (Koenen et al., 2015; Heads, 2019), agrupadas en cuatro subfamilias: Melioideae, la más grande integrada por siete tribus y 35 géneros; Swietenioideae dividida en tres tribus y 13 géneros, y Quivisianthoideae y Capuronianthoideae, con solo un género respectivamente (Pennington y Styles, 1975). Análisis filogenéticos soportan la monofilia de la familia; solo las subfamilias Melioideae y Swietenioideae son reconocidas, mientras que Quivisianthoideae y Capuronianthoideae se anidan en Melioideae y Swietenioideae, respectivamente (Muellner et al., 2003; Koenen et al., 2015). En América se pueden encontrar siete géneros y 126 especies con distribución natural, así como siete géneros introducidos: Aphanamixis Blume, Azadirachta A. Juss., Chukrasia A. Juss., Melia L., Khaya, Sandoricum Cav. y Toona (Endl.) M. Roem., todos ellos de uso ornamental (Germán-Ramírez, 2005). En México se distribuyen cinco géneros y 24 especies nativas, lo cual se concluye después de un análisis nomenclatural de las especies citadas por Germán-Ramírez (2005) y Villaseñor (2016).

En una revisión de ejemplares de Meliaceae colectadas en Tabasco, depositadas en los herbarios CSAT y UJAT, se observó que la gran mayoría corresponden a tres especies (Trichilia cuneata Radlk., T. havanensis Jacq. y Cedrela odorata), lo que evidenció la falta de trabajo de campo en la colecta de material botánico de otras pobremente representadas, haciéndose notar que muchos ejemplares depositados en los dos herbarios y mencionados en diferentes trabajos florísticos son nombrados por sus sinónimos o bien con determinaciones erróneas. Por lo tanto, esta contribución como parte del proyecto "Flora de Tabasco" enlista las especies de la familia con distribución en el estado. Así mismo, se generó una clave dicotómica para las especies, además de reunir información de la dis- 
tribución geográfica y ecológica de la familia Meliaceae en Tabasco. Aunque el estudio está restringido a un área política delimitada, el análisis de las especies encontradas contribuirá a documentar la variabilidad de los taxa distribuidos en el estado y brindará elementos descriptivos de la variabilidad de los caracteres que puede ser utilizada en trabajos similares en áreas de mayor cobertura geográfica.

\section{Materiales y Métodos}

\section{Área de estudio}

El estado de Tabasco cuenta con una extensión de 24,475 $\mathrm{km}^{2}$ dividido en 17 municipios. Está situado entre los paralelos $18^{\circ} 39^{\prime} 03^{\prime \prime}$ y $17^{\circ} 15^{\prime} 03^{\prime \prime}$ de latitud norte y los meridianos $94^{\circ} 07^{\prime} 48^{\prime \prime}$ y $90^{\circ} 59^{\prime} 15^{\prime \prime}$ de longitud oeste, limita al norte con el Golfo de México y el estado de Campeche, al sur con el estado de Chiapas, al este con Veracruz, al noreste con Campeche y al sureste con la República de Guatemala (Fig. 1). Tabasco se ubica en la provincia denominada Llanura Costera del Golfo Sur. Presenta un relieve plano en la mayoría de su territorio; hacia el sur se localiza una porción de sierras o montañas localizada en la parte baja de la vertiente de la Sierra Madre Oriental, la cual pertenece a la provincia de Sierras de Chiapas y Guatemala, cuyas altitudes se ubican entre 40 y 1100 m. Los tipos de clima que prevalecen en la entidad, siguiendo la clasificación de Köppen modificada por García (2004), son el Am(f) que se distribuye de la costa en toda la llanura y el $\mathrm{Af}(\mathrm{m})$ que abarca la zona de la sierra. La temperatura media anual es de alrededor de $27^{\circ} \mathrm{C}$. La precipitación pluvial media anual es de unos $2550 \mathrm{~mm}$ y se va incrementando gradualmente hacia el sur, donde se registra una media anual de 4000 mm en la zona de la sierra (INEGI, 2017). También existe un periodo de sequía, conocido como "canícula" que se presenta a finales de julio hasta la primera mitad de agosto. Las precipitaciones de invierno son mayormente provocadas por la llegada de los fuertes fríos o "nortes", comunes desde noviembre hasta febrero. Los ciclones y huracanes tropicales del Golfo de México se manifiestan entre junio y octubre, afectando una amplia extensión territorial (Salazar-Tosca, 1994).

Los tipos de vegetación de acuerdo con Rzedowski (2006) presentes en el estado son: 1) Bosque tropical perennifolio, 2) Bosque espinoso, 3) Pastizal, 4) Bosque mesófilo de montaña, 5) Vegetación acuática y subacuática (manglar, popal, tular y carrizal, vegetación flotante, vegetación sumergida, bosque de galería) y 6) Otros tipos de vegetación (bosque de Byrsonima Rich. ex Kunth, $\mathrm{Cu}$ ratella Loefl. y Crescentia L., palmar, vegetación halófita).

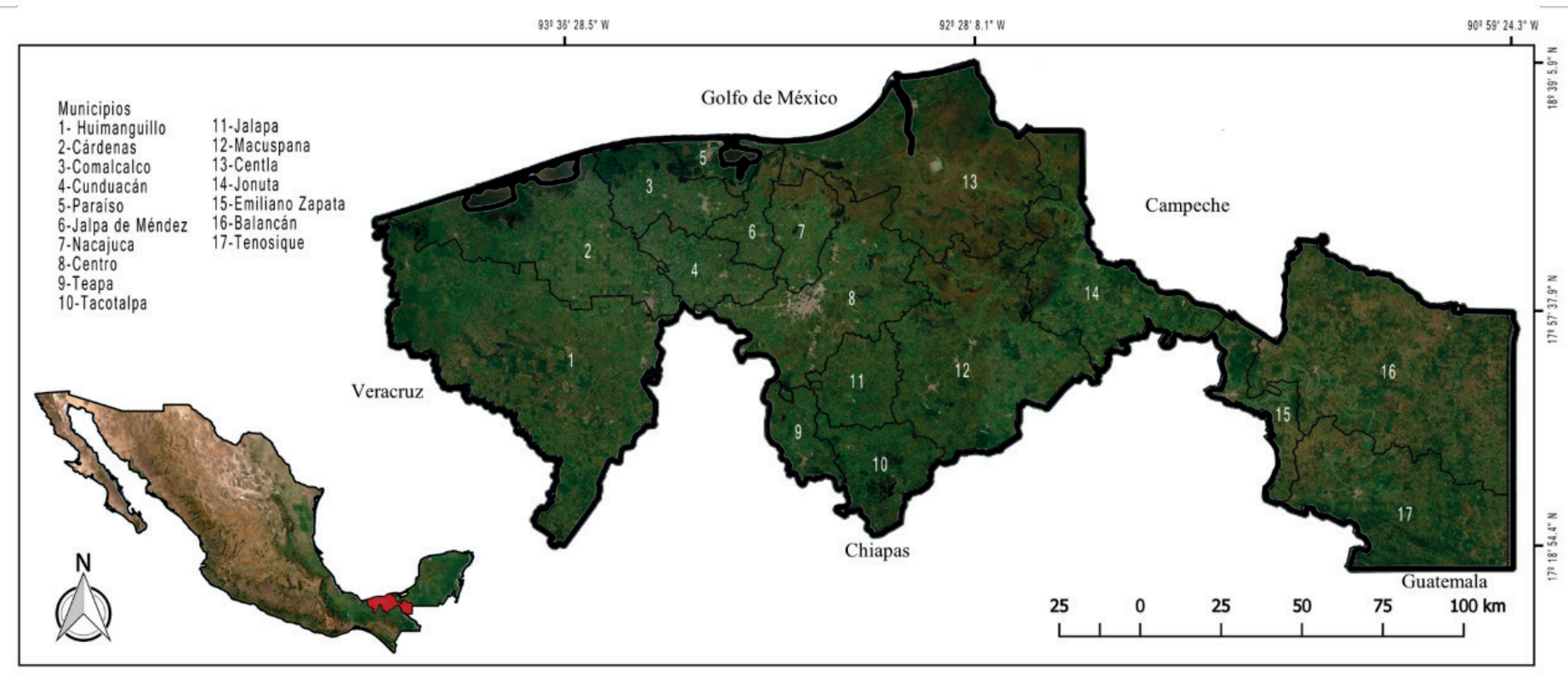

Figura 1: Ubicación del estado de Tabasco en México y su división política. 
Todos los tipos de vegetación han sufrido afectaciones antropogénicas de diferentes magnitudes, documentándose principalmente la pérdida de las selvas, las cuales en 1950 se extendían en poco más de medio millón de hectáreas y para 1990 sus remanentes ocupaban menos de cincuenta mil hectáreas, localizadas en manchones de diferentes tamaños en los municipios de la serranía como Huimanguillo, Macuspana, Teapa, Tenosique y Tacotalpa (Sánchez-Munguía, 2005).

Colecta de material botánico y revisión de herbarios

Para el desarrollo de este trabajo se realizaron colectas durante los años 2015 a 2017, en todos los municipios y tipos de vegetación de Tabasco, siguiendo la metodología recomendada por Lot y Chiang (1986). De cada ejemplar colectado se tomaron datos de la localidad (nombre, municipio, coordenadas geográficas y tipo de vegetación), así como las características biológicas que ayudaron a su determinación (forma de vida, altura, color de flor, forma y tipo del fruto, semillas). De igual manera se revisaron los herbarios CSAT (Colegio de Postgraduados, campus Tabasco), MEXU (Universidad Nacional Autónoma de México), UJAT (Universidad Juárez Autónoma de México) y XAL (Instituto de Ecología A.C.), donde se corroboró la determinación y se obtuvieron los datos contenidos en la etiqueta de los ejemplares de Tabasco. La información de los ejemplares colectados y de los revisados en los herbarios se organizaron y analizaron para cada especie.

\section{Identificación de las especies de Meliaceae}

La identificación se realizó utilizando las claves de los trabajos florísticos de Guatemala (Standley y Steyermark, 1946), Flora de Panamá (Smith, 1965), Flora de Nicaragua (Pennington y Styles, 2001) y las monografías de Meliaceae (Pennington et al., 1981) y de los géneros Trichilia P. Browne (Pennington, 2016) y Guarea F. Allam. ex L. (Pennington y Clarkson, 2013), así como el análisis del complejo de Guarea glabra Vahl (Coronado, 2006). Los ejemplares fueron montados e incluidos en la colección del herbario UJAT y sus duplicados fueron enviados a herbarios nacionales (MEXU y XAL). Del análisis de los caracteres de las flores y frutos principalmente, y en menor grado de las hojas, se elaboró la clave dicotómica de identificación para las especies de Meliaceae de Tabasco.

\section{Resultados}

En Tabasco la familia Meliaceae está representada por cuatro géneros y 11 especies nativas, así como dos géneros y dos especies introducidas (Cuadro 1), de las cuales Trichilia trifolia L. y T. breviflora Blake \& Standl. son nuevos registros para el estado. El género con mayor diversidad de especies es Trichilia con siete especies, seguido de Guarea con dos y el resto de los géneros con una sola. Azadirachta indica A. Juss. y Melia azedarach L. son introducidas y cultivadas con fines de interés ornamental y las especies nativas Cedrela odorata y Swietenia macrophylla son cultivadas con fines maderables, por lo que estas cuatro especies se encuentran ampliamente distribuidas en todo el territorio estatal. En cuanto a su distribución, el mayor número de especies se encuentra en vegetación secundaria de bosque tropical perennifolio donde se distribuyen seis especies, seguido del bosque mediano subcaducifolio con cinco especies, el bosque tropical perennifolio, el pastizal cultivado y los huertos familiares con cuatro cada una; tres se distribuyen en la vegetación riparia, mientras que en los cercos vivos, la vegetación secundaria de bosque mediano subcaducifolio y jardines se encuentran dos. La distribución de especies por municipio es de la siguiente manera: Teapa y Centro presentan el mayor número con ocho; seguido de Balancán con siete, Comalcalco y Tenosique con cuatro, Cunduacán, Huimanguillo, Macuspana, Nacajuca y Tacotalpa con tres, Cárdenas, Centla, Emiliano Zapata, Jalapa, Jalpa de Méndez y Paraíso con dos y Jonuta con una (Cuadro 1, Fig. 2, Apéndice).

Algunas de las especies se ilustran en las figuras 3-7. Del análisis de caracteres y estados de carácter de flores, frutos, semillas y hojas fueron utilizados principalmente para estructurar la clave de identificación para las especies de la familia Meliaceae en Tabasco.

Clave para la identificación de las especies de Meliaceae en Tabasco

1a. Fruto una drupa; plantas cultivadas, introducidas .......... 2

1a. Fruto una cápsula; plantas nativas . 3

2a. Hojas bipinnadas; pétalos blanco-rosa a violeta Melia azedarach $\mathrm{L}$.

2b. Hojas una vez pinnadas; pétalos blancos a blancos-verdosos Azadirachta indica A. Juss. (Fig. 3)

3a. Semillas aladas, sin arilo en semillas maduras 4 
3b. Semillas no aladas, con arilo naranja o rojizo en semillas maduras 5

4a. Estambres 5, libres entre sí, adnados en su parte inferior a un androginóforo, anteras adheridas en el ápice de los filamentos Cedrela odorata L. (Fig. 4)

4b. Estambres 8-10, filamentos completamente connados en un tubo, anteras adheridas dentro de la garganta del tubo Swietenia macrophylla King (Fig. 5)

5a. Anteras adheridas dentro de la garganta del tubo estaminal; hojas con una yema terminal con crecimiento indeterminado

5b. Anteras adheridas en el ápice de los filamentos o en el margen del tubo estaminal; hojas sin crecimiento indeterminado

7

6a. Pétalos 4-4.5 $\mathrm{mm}$ de largo; frutos con 2-4 valvas, valvas 1.5-4 cm de largo, glabras ... Guarea glabra Vahl. (Fig. 6) 6b. Pétalos 9-17 mm de largo; frutos con 5-8 valvas, valvas
3.5-8 cm de largo, densamente puberosas o papilosas Guarea grandifolia DC.

7b. Hojas trifoliadas Trichilia trifolia $\mathrm{L}$.

7a. Hojas unifoliadas o pinnadas ............................................ 8

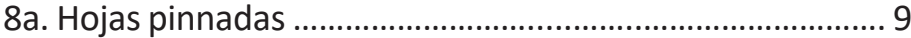

8b. Hojas unifoliadas ......... Trichilia breviflora Blake \& Standl.

9a. Pecíolo y raquis alados ... Trichilia havanensis Jacq. (Fig. 7)

9a. Pecíolo y raquis no alados 10

10a. Inflorescencias $1-3 \mathrm{~cm}$ de largo, fasciculadas Trichilia pallida Sw.

10b. Inflorescencias más de $3 \mathrm{~cm}$ de largo, tirsoides 11

11a. Pétalos usualmente glabros ....... Trichilia hirta L. (Fig. 7)

11b. Pétalos frecuentemente puberulentos 12

12a. Fruto $1-1.3 \mathrm{~cm}$ de largo, glandular papiloso con pelos cortos Trichilia martiana C. DC. (Fig. 7)

12b. Fruto $1.6-2.4 \mathrm{~cm}$ de largo, liso, seríceo a tomentoso ...... Trichilia moschata Sw.

Cuadro 1: Especies de la familia Meliaceae y distribución por municipio y tipo de vegetación en el estado de Tabasco, México: Cerco vivo=Cv, Huerto familiar=Hf, Jardines=Jar, Pastizal cultivado=Pc, Bosque tropical perennifolio=Btp, Bosque mediano subcaducifolio= Bms, Vegetación secundaria de bosque tropical perennifolio=Vbtp, Vegetación secundaria de bosque mediano subcaducifolio=Vbms, Vegetación riparia=Vri. * =Especie introducida.

\begin{tabular}{|c|c|c|c|}
\hline Especie & Nombre común & Municipios & Vegetación \\
\hline Azadirachta indica A. Juss* & Neem & Balancán, Centro, Emiliano Zapata, Paraíso & $\mathrm{Cv}, \mathrm{Hf}, \mathrm{Jar}, \mathrm{Pc}$ \\
\hline Cedrela odorata $\mathrm{L}$. & Cedro & $\begin{array}{l}\text { Balancán, Centro, Comalcalco, Jalapa, Macuspana, } \\
\text { Nacajuca, Teapa }\end{array}$ & $\mathrm{Hf}, \mathrm{Pc}, \mathrm{Bms}, \mathrm{Vbtp}$ \\
\hline Guarea glabra Vahl. & $\begin{array}{l}\text { Chichón de árbol, } \\
\text { tomatillo }\end{array}$ & $\begin{array}{l}\text { Balancán, Centro, Centla, Huimanguillo, Jonuta, } \\
\text { Macuspana, Tacotalpa, Teapa, Tenosique }\end{array}$ & Btp, Bms, Vbtp \\
\hline Guarea grandifolia DC. & $\begin{array}{l}\text { Castarrica, quebracho } \\
\text { negro }\end{array}$ & Centro, Teapa, Tenosique & Bms \\
\hline Melia azedarach L.* & Paraíso & $\begin{array}{l}\text { Centla, Comalcalco, Cunduacán, Huimanguillo, Jalapa, } \\
\text { Nacajuca }\end{array}$ & $\mathrm{Hf}$, Jar \\
\hline Swietenia macrophylla King. & Caoba & Balancán, Cárdenas, Centro, Teapa & Hf, Pc, Btp, Bms, Vbtp \\
\hline Trichilia breviflora Blake \& Standl. & & Teapa & Vri \\
\hline Trichilia havanensis Jacq. & Coshihua, cedrillo & $\begin{array}{l}\text { Balancán, Cárdenas, Centro, Comalcalco, Cunduacán, } \\
\text { Jalpa de Méndez, Paraíso, Tacotalpa, Teapa, Tenosique }\end{array}$ & Smsc, Vbtp, Vbms, Vri \\
\hline Trichilia hirta $\mathrm{L}$. & & $\begin{array}{l}\text { Balancán, Centro, Cunduacán, Emiliano Zapata, Teapa, } \\
\text { Tenosique }\end{array}$ & Cv, Pc, Btp, Vri \\
\hline Trichilia martiana C. DC. & & $\begin{array}{l}\text { Centro, Comalcalco, Macuspana, Nacajuca, Tacotalpa, } \\
\text { Teapa }\end{array}$ & Vbtp \\
\hline Trichilia moschata Sw. & & Jalpa de Méndez & Bms \\
\hline Trichilia pallida Sw. & & Huimanguillo & Btp \\
\hline Trichilia trifolia $\mathrm{L}$. & & Balancán & Vbtp, Vbms \\
\hline
\end{tabular}




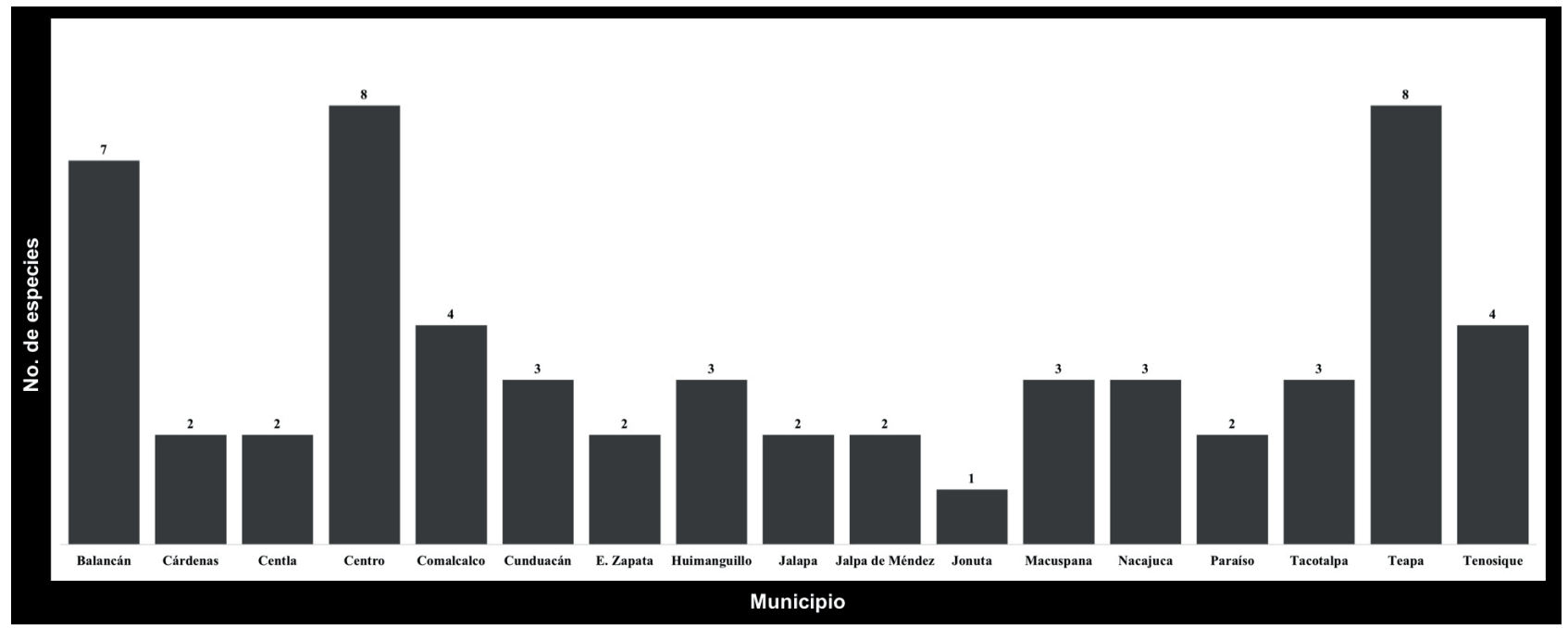

Figura 2: Número de especies y distribución por municipio de la familia Meliaceae en el estado de Tabasco, México.

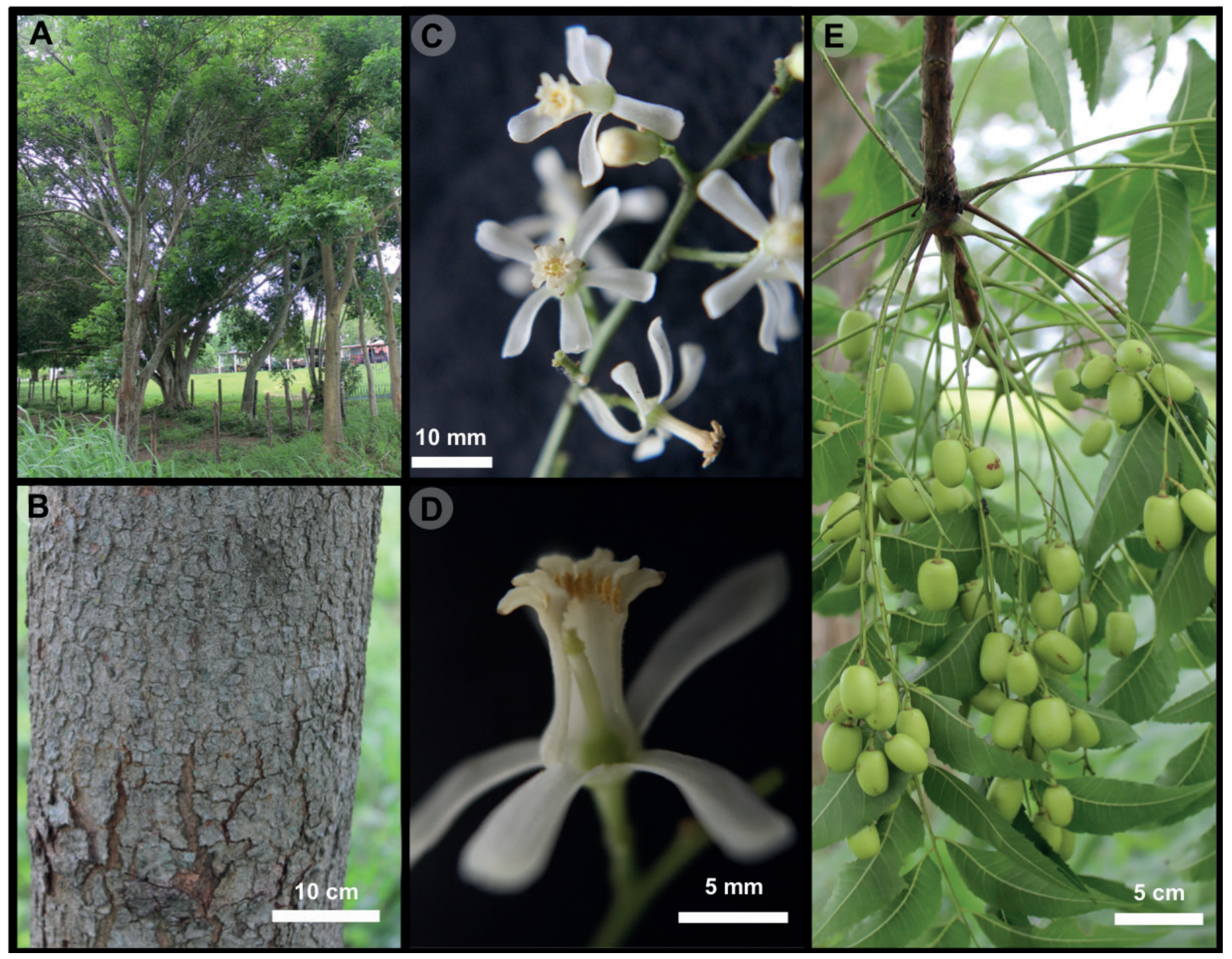

Figura 3: Azadirachta indica A. Juss. A. individuos usados como cerco vivo; B. detalle del tallo; C. detalle de la flor; D. vista interna en corte longitudinal de la flor; E. frutos inmaduros. 


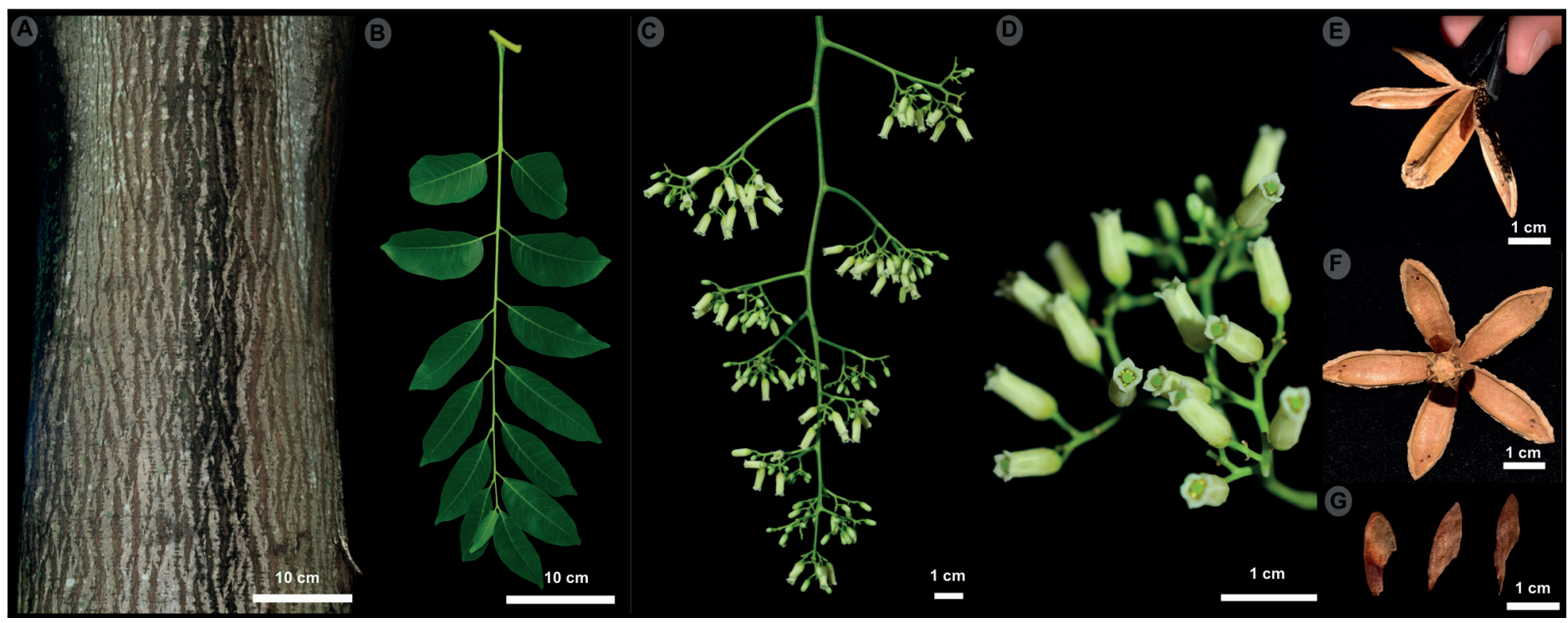

Figura 4: Cedrela odorata L. A. detalle de la corteza; B. detalle de la hoja; C. inflorescencia; D. detalle de las flores; E-F. frutos dehiscentes; G. semillas.

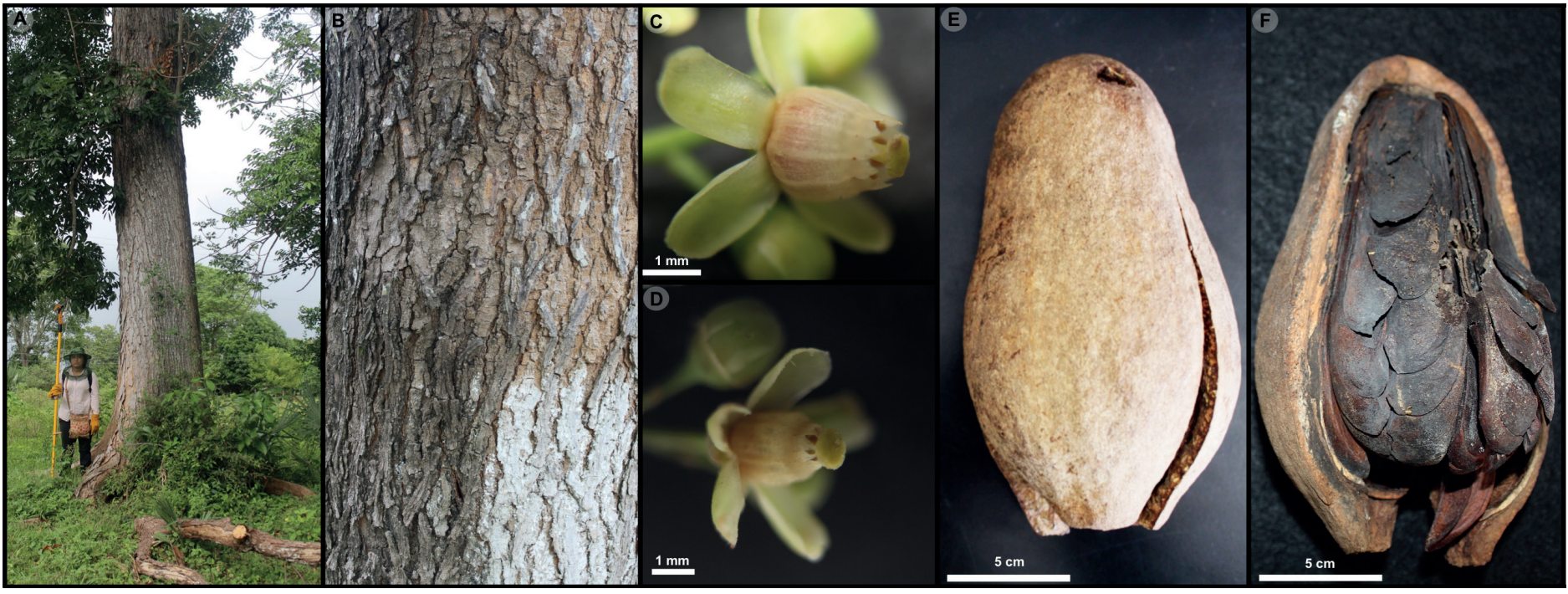

Figura 5: Swietenia macrophylla King. A-B. vista del fuste y detalle de la corteza; C-D. detalle de la flor; E. vista del fruto; F. vista del fruto, mostrando las semillas.

\section{Discusión}

El presente trabajo representa un primer acercamiento a la diversidad de la familia Meliaceae en su actual circunscripción taxonómica y bajo un tratamiento taxonómico en el sureste de México, donde es un componente importante, tanto en estructura como en número de especies en los remanentes de los diferentes estratos del bosque tropical perennifolio a lo largo del país (Pennington y Sarukhán, 2005).
De las 24 especies nativas presentes en México (Pennington et al., 1981; Calderón y Germán-Ramírez, 1993; Germán-Ramírez, 2005; Penninghton y Clarkson, 2013; Pennington, 2016), 11 se encuentran en Tabasco, mientras que las 13 restantes se distribuyen en tipos de vegetación no presentes en el estado o en áreas biogeográficas diferentes (Cuadro 2). La ausencia de algunas, a pesar de ser reportadas en estados vecinos y en tipos de vegetación 


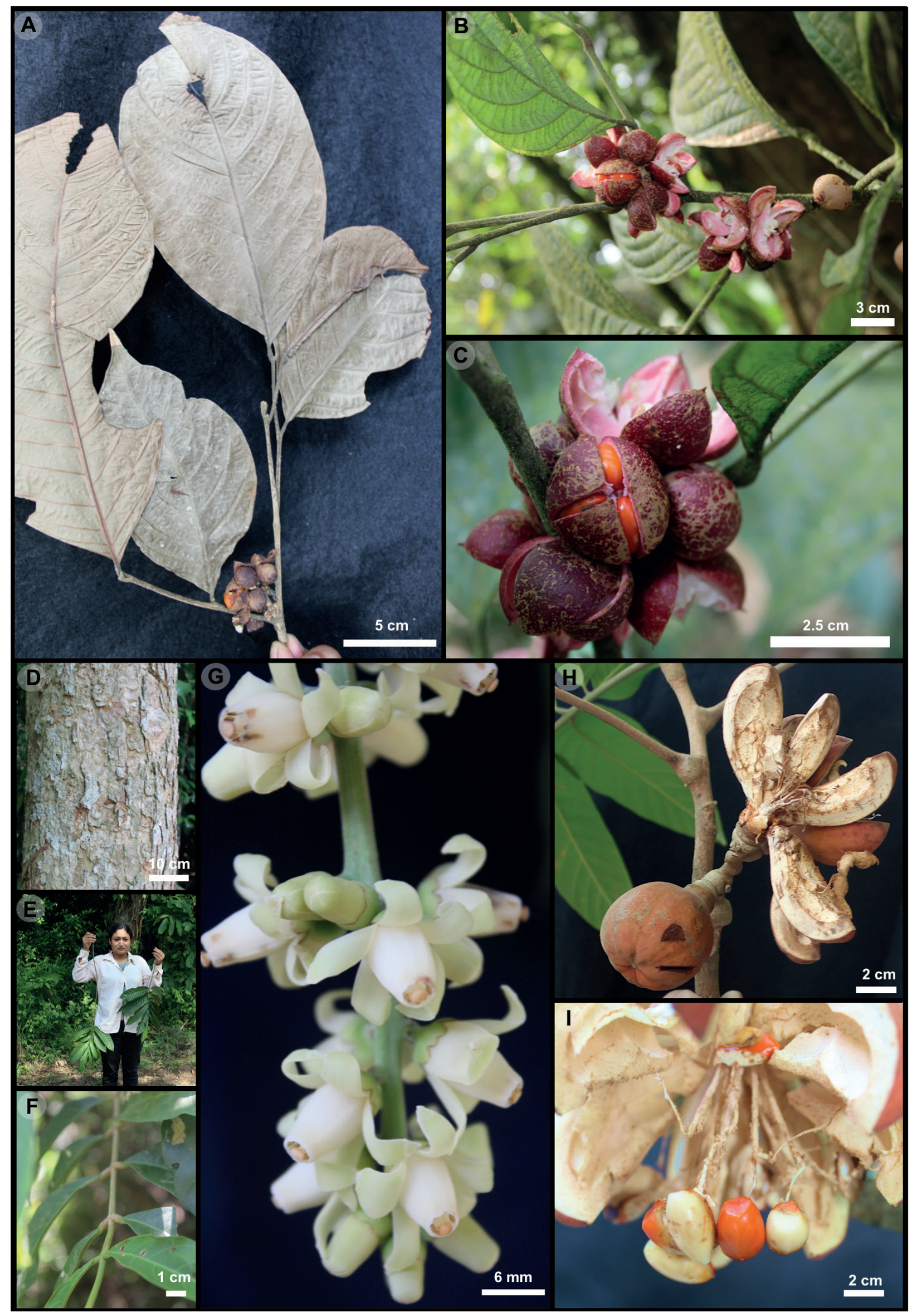

Figura 6: Guarea glabra Vahl. A. detalle de la hoja; B-C. frutos y semillas. G. grandifolia DC. D. detalle de la corteza; E-F. vista general y en detalle de la hoja; G. flores; H. frutos dehiscentes; I. detalle del fruto y semillas. 


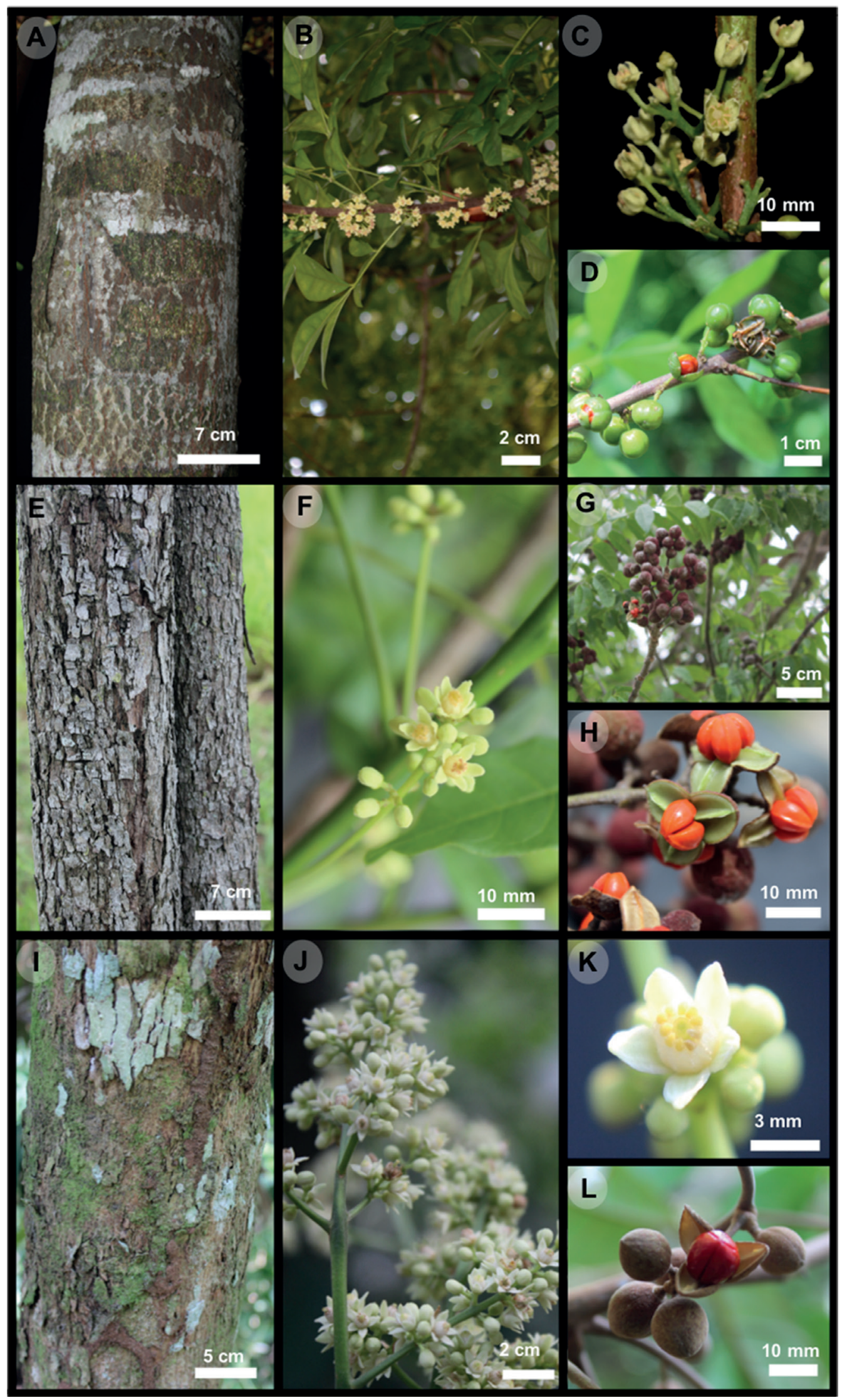

Figura 7: Trichilia havanensis Jacq. A. detalle del tallo; B. ramas con inflorescencias; C. flores; D. frutos dehiscentes mostrando las semillas. T. hirta L. E. detalle de la corteza; F. flores; G-H. frutos dehiscentes y semillas. T. martiana C. DC. I. detalle del tallo; J. vista de la inflorescencia; K. detalle de la flor; L. frutos y semillas frescas. 
compartidos, puede ser debido a que en Tabasco el bosque tropical perennifolio ha sido destruido básicamente por el cambio de uso de suelo, el crecimiento poblacional, la ganadería, construcción de carreteras, extracción de madera y actividades petroleras (Gómez-Pompa, 1990; SalazarConde et al., 2004; Guerra-Martínez y Ochoa-Gaona, 2008), además de incendios naturales o intencionales, que en conjunto ocasionan una pérdida de 2283 ha en promedio de selva al año (Sánchez-Munguía, 2005). Todo esto ha tenido como consecuencia que la vegetación del estado sea en su mayoría secundaria. Por lo tanto, es muy probable que en Tabasco se haya llevado un proceso de extirpación local de varias especies de Meliáceas y aunque los estados vecinos igualmente han sufrido perdida de las selvas, en éstos, se extienden en mayores áreas y ambientes, sobre todo existen áreas protegidas que han mantenido amplios manchones de selva sin alteraciones considerables, cosa que no sucede en Tabasco. Una observación que permite considerar lo anterior es la distribución de las especies de Meliaceae en Tabasco, donde nueve especies que representan $81 \%$ de las nativas se encuentran concentradas en regiones con vegetación secundaria de los diferentes tipos de bosque tropical principalmente, donde los géneros Guarea y Trichilia son importantes elementos estructurales y en la vegetación conservada de selva en zonas generalmente poco accesibles de los municipios serranos.
Es interesante notar que de las especies Cedrela odorata y Swietenia macrophylla no se lograron obtener colectas en zonas de vegetación primaria (aunque guías y pobladores locales mencionan que sigue siendo común en la "montaña"), ocasionado por la extracción masiva de estas especies desde mediados del siglo XIX (West et al., 1985; Capdepont-Ballina, 2008), llegándose a considerar como saqueo de recursos maderables para el mercado anglosajon (Rangel-González, 2014). Aunque no existen datos de los volúmenes de madera extraída, queda claro que esta sobreexplotación generó cambios en la cubertura forestal de la entidad (Vargas-Simón et al., 2019). Actualmente, estas mismas especies son ampliamente distribuidas en el estado por su uso en campañas de reforestación, en plantaciones forestales y como plantas ornamentales en jardines.

\section{Contribución de autores}

DCC y CBR desarrollaron y diseñaron el proyecto de investigación, realizaron la colecta en campo y consultaron los ejemplares de herbario, la descripción de las especies y las claves dicotómicas. NMR, MCD, WAJ, GBS y MOR contribuyeron en la colecta en campo, descripción de las especies y la elaboración de figuras. NMC apoyó en la colecta y se encargó del procesamiento, montaje e intercalado de los ejemplares. Todos los autores contribuyeron a la redacción, discusión, revisión y aprobación del manuscrito final.

Cuadro 2: Distribución en México de las especies nativas no presentes en Tabasco (Calderón y Germán-Ramírez, 1993; Germán-Ramírez, 2005; Pennington et al., 1981; Pennington y Clarkson, 2013; Pennington, 2016).

\begin{tabular}{ll}
\hline Especie & Distribución \\
\hline Cedrela angustifolia DC. & Michoacán, Sinaloa y Veracruz. Endémica de México \\
Cedrela discolor S.F. Blake & Durango y Guerrero. Endémica de México \\
Cedrela dugesii S.Watson & Michoacán, Guanajuato, Jalisco y Querétaro. Endémica de México \\
Cedrela oaxaquensis C. DC. \& Rose & Guerrero, Estado de México, Morelos, Puebla y Oaxaca. Endémica de México \\
Cedrela salvadorensis Standl. & Chiapas, Guerrero, Jalisco, México, Michoacán, Morelos, Oaxaca y Puebla. \\
Cedrela tonduzii C. DC. & Chiapas, Oaxaca \\
Guarea mexicana Coronado & Chiapas. \\
Swietenia humilis Zucc. & Sinaloa y Durango a través de la vertiente del Pacífico hasta Chiapas \\
Trichilia americana (Sessé \& Moc.) T.D. Penn & Chihuahua, Sonora a través de la vertiente del Pacífico de México. \\
Trichilia chirriactensis (Standl. \& Steyerm.) T.D. Penn. & Chiapas \\
Trichilia erythrocarpa Lundell & Chiapas, Oaxaca y Veracruz \\
Trichilia glabra L. & Península de Yucatán \\
Trichilia minutiflora Standl. & Chiapas, Campeche y Quintana Roo \\
\hline
\end{tabular}




\section{Financiamiento}

Este estudio fue apoyado por la Universidad Juárez Autónoma de Tabasco mediante el Programa de Fortalecimiento a la Investigación que financió el proyecto UJAT-2014-PFIE-01 otorgado a GBS, así como por recursos propios de DCC y CBR.

\section{Agradecimientos}

Los autores agradecen a los curadores de los herbarios quienes permitieron la revisión de sus colecciones. A los revisores anónimos que con sus valiosos comentarios enriquecieron el trabajo y a Germán Carnevalli FernándezConcha por su ayuda y sugerencias al manuscrito.

\section{Literatura citada}

APG IV. 2016. An update of the Angiosperm Phylogeny Group classification for the orders and families of flowering plants: APG IV. Botanical Journal of the Linnean Society 181(1): 1-20. DOI: https://doi.org/10.1111/boj.12385

Calderón, G. y M. T. Germán-Ramírez. 1993. Meliaceae. Flora del Bajío y de Regiones Adyacentes 11: 1-22.

Capdepont-Ballina, J. L. 2008. Con la furia de las sierras cayeron las caobas y se fue nadando la selva. Las monterías en las sierras de Tabasco y Chiapas (1855-1936). Tesis de Doctorado en Historia. Centro de Estudios Históricos. El Colegio de Michoacán, A.C. Zamora, Michoacán, México. 422 pp.

CITES. 2019. Appendices I, II, II. Convention on international trade in endangered species of wild fauna and flora. https:// cites.org/sites/default/files/eng/app/2019/E-Appendices-2019-11-26.pdf (consultado julio de 2020).

Cornelius, J. P., C. M. Navarro, K. E. Wightman y S. E. Ward. 2005. Is mahogany dysgenically selected? Environmental Conservation 32(2): 129-139. DOI: https://doi.org/10.1017/ S0376892905002158

Coronado, I. M. 2006. Five new species of Guarea (Meliaceae), Two from the Guarea glabra Vahl complex, in Central America. Novon: A Journal for Botanical Nomenclature 16(4): 462-467. DOI: https://doi.org/10.3417/10553177(2006)16[462:FNSOGM]2.0.CO;2

García, E. 2004. Modificaciones al sistema de clasificación climática de Köppen. $5^{\text {a }}$ Ed. Instituto de Geografía. Universidad Nacional Autónoma de México. México, D.F., México. 90 pp.

Germán-Ramírez, M. T. 2005. Meliaceae. In: Novelo-Retana, A. (ed.). Flora del Valle de Tehuacán-Cuicat- lán. Instituto de Biología. Universidad Nacional Autónoma de México. México, D.F., México. 42: 1-15. http://www.ibiologia.unam.mx/BIBLIO68/fulltext/ fasiculosfloras/f42_meli.pdf (consultado abril de 2020).

Gómez-Pompa, A. 1990. El problema de la deforestación en el trópico mexicano. In: Leff, E. (ed.). Medio Ambiente y Desarrollo en México. Volumen I. Centro de Investigaciones Interdisciplinarias en Ciencias y Humanidades. Universidad Nacional Autónoma de México y Editorial Porrúa. México, D.F., México. Pp. 229-255.

Guerra-Martínez, V. y S. Ochoa-Gaona. 2008. Evaluación del programa de manejo de la Reserva de la Biosfera Pantanos de Centla en Tabasco, México. Universidad y Ciencia 24(2): 135-146.

Gullison, R. E., R. E. Rice y A. G. Blundell. 2000. 'Marketing' species conservation. Nature 404(6781): 923-924. DOI: https:// doi.org/10.1038/35010151

Heads, M. 2019. Biogeography and ecology in a pantropical family, the Meliaceae. Gardens' Bulletin Singapore 71(Suppl. 2): 335-461. DOI: https://doi.org/10.26492/gbs71(suppl.2).2019-22

INEGI. 2017. Síntesis geográfica de Tabasco. Instituto Nacional de Estadística Geografía e Informática. Secretaría de Programación y Presupuesto. México, D.F., México. 443 pp.

IUCN. 2020. The International Union for Conservation of $\mathrm{Na}$ ture's. Red List of Threatened Species. Version 2020-1. http://www.iucnredlist.org/search?page=5 (consultado abril de 2020).

Koenen, E. J. M., J. J. Clarkson, T. D. Pennington y L. W. Chatrou. 2015. Recently evolved diversity and convergent radiations of rainforest mahoganies (Meliaceae) shed new light on the origins of rainforest hyperdiversity. New Phytolist 207(2): 327-339. DOI: https://doi.org/10.1111/nph.13490

Kometter, R. F., M. Martínez, A. G. Blundell, R. E. Gullison, M. K. Steininger y R. E. Rice. 2004. Impacts of unsustainable mahogany logging in Bolivia and Peru. Ecology and Society 9(1): 12. http://www.ecologyandsociety.org/vol9/iss1/ art12 (consultado julio de 2020).

Laurance, W. F. 1999. Reflections on the tropical deforestation crisis. Biological Conservation 91(2-3): 109-117. DOI: https:// doi.org/10.1016/S0006-3207(99)00088-9

López-Camacho, R. y D. Cárdenas-López. 2002. Manual de identificación de especies maderables objeto de comercio en 
la Amazonia colombiana. Instituto Amazónico de Investigaciones Científicas, SINCHI. Bogotá, D.C., Colombia. 100 pp. https://sinchi.org.co/files/publicaciones/publicaciones/pdf/ ManualMaderas.pdf (consultado julio de 2020).

Lot, A. y F. Chiang. 1986. Manual de herbario: Administración y manejo de colecciones, técnicas de recolección y preparación de ejemplares botánicos. Departamento de Botánica, Instituto de Biología. Universidad Nacional Autónoma de México. Consejo nacional de flora de México A. C. México, D.F., México. 342 pp.

Muellner, A. N., R. Samuel, S. A. Johnson, M. Cheek, T. D. Pennington y M. W. Chase. 2003. Molecular phylogenetics of Meliaceae (Sapindales) based on nuclear and plastid DNA sequences. American Journal of Botany 90(3): 471-480. DOI: https://doi. org/10.3732/ajb.90.3.471

O’Neill, G. A., I. Dawson, C. Sotelo-Montes, L. Guarino, M. Guariguata, D. Current y J. C. Weber. 2001. Strategies for genetic conservation of trees in the Peruvian Amazon. Biodiversity and Conservation 10: 837-850. DOI: https://doi. org/10.1023/A:1016644706237

Pennington, T. D. 2016. Systematic treatment of American Trichilia (Meliaceae). Phytotaxa 259(1): 018-162. DOI: https://doi. org/10.11646/phytotaxa.259.1.5

Pennington, T. D. y J. J. Clarkson. 2013. A revision of Guarea (Meliaceae). Trustees of the Royal Botanic Garden Edinburgh. Edinburgh Journal of Botany 70(2): 179-362. DOI: https://doi. org/10.1017/S0960428613000036

Pennington T. D. y J. Sarukhán. 2005. Árboles tropicales de México: Manual para la identificación de las principales especies. Universidad Nacional Autónoma de México. Fondo de Cultura Económica. México, D.F., México. 523 pp.

Pennington, T. D. y B. T. Styles. 1975. A generic monograph of the Meliaceae. Blumea 22(3): 419-540.

Pennington, T. D. y B. T. Styles. 2001. Meliaceae. In: Stevens, W. D., C. Ulloa, A. Pool y O. Montiel (eds.). Flora de Nicaragua. Tome II. Monographs in Systematic Botany from the Missouri Botanical Garden 85: 1419-1430.

Pennington, T. D., B. T. Styles y D. H. Taylor. 1981. Meliaceae with Accounts of Swietenioideae and Chemotaxonomy. Flora Neotropica Monograph 28: 1-470.

Rangel-González, E. J. 2014. Compañías deslindadoras sociedades forestales. Empresariado en el entorno fronterizo de la Cos- ta Oriental y creación de un borde en las márgenes de Río Hondo, 1876-1935. Tesis de doctorado en Historia. Centro de Investigaciones y estudios Superiores en Antropología Social. Mérida, México. 430 pp.

Rodan, B. D., A. C. Newton y A. Veríssimo. 1992. Mahogany conservation: status and policy initiatives. Enviromental Conservation 19(4): 331-338. DOI: https://doi.org/10.1017/ S0376892900031453

Rzedowski, J. 2006. Vegetación de México. $1^{\text {a }}$ edición digital. Comisión Nacional para el Conocimiento y Uso de la Biodiversidad (CONABIO). México, D.F., México. 504 pp.

Salazar-Conde, E. C., J. Zavala-Cruz, O. Castillo-Acosta y R. CámaraArtigas. 2004. Evaluación espacial y temporal de la vegetación de la Sierra Madrigal, Tabasco, México (1973-2003). Investigaciones Geográficas 54: 7-23.

Salazar-Tosca, J. C. 1994. Compendio monográfico de la historia de Tabasco: Obra literaria pedagógica. 2a Edición. Villahermosa, Tabasco, México. 256 pp.

Sánchez-Munguía, A. 2005. Uso del Suelo Agropecuario y Desforestación en Tabasco 1950-2000. Universidad Juárez Autónoma de Tabasco. Colección José Narcizo Rovirosa. Villahermosa, Tabasco, México. 129 pp.

Smith, C. E. 1965. Meliaceae: Flora de Panamá. Annals of the Missouri Botanical Garden 52: 55-79.

Standley, C. P. y A. J. Steyermark. 1946. Meliaceae: Flora de Guatemala. Fieldiana Botany 24(5): 444-468.

Thiago, A., M. R. Lemes, J. Grogan y R. Gribel. 2008. Post-logging loss of genetic diversity in a mahogany (Swietenia macrophylla King, Meliaceae) population in Brazilian Amazonia. Forest Ecology Management 255(2): 340-345. DOI: https://doi. org/10.1016/j.foreco.2007.09.055

Vargas-Simón, G., L. F. Zamora-Cornelio, B. Morales-García, P. DíazJiménez, C. Tovilla-Hernández, C. E. Zenteno-Ruíz y L. D. Olivera-Gómez. 2019. Sobreexplotación de la Biodiversidad. In: Comisión Nacional para el Conocimiento y Uso de la Biodiversidad (CONABIO) (ed.). La biodiversidad en Tabasco: Estudio de Estado. Vol. III. Gobierno del Estado de Tabasco, Universidad Juárez Autónoma de Tabasco. Colegio de Posgraduados. México, D.F., México. 135-142 pp.

Veríssimo, A., C. J. Souza, S. Stone y C. Uhl. 1998. Zoning of timber extraction in the Brazilian Amazon. Conservation Biology 12(1): 128-136. DOI: https://doi.org/10.1111/j.15231739.1998.96234.x 
Villaseñor, J. L. 2016. Checklist of the native vascular plants of Mexico. Revista Mexicana de Biodiversidad 87(3): 559-902.

DOI: https://doi.org/10.1016/j.rmb.2016.06.017
West, R. C., N. P. Psuty y B. G. Thom. 1985. Las tierras bajas de Tabasco en el sureste de México. Gobierno del Estado de Tabasco. México, D.F., México. 409 pp. 
Apéndice: Ejemplares examinados de la familia Meliaceae por municipio en el estado de Tabasco, México. *=Especie introducida.

Azadirachta indica A. Juss. Municipio: Balancán, D. Córdova-Córdova 134 (UJAT). Municipio: Centro, E. Chávez 22 (UJAT); D. Córdova-Córdova 01 (UJAT); E. López 345 (UJAT). Municipio: Emiliano Zapata, D. Córdova-Córdova et al. 34 (UJAT). Municipio: Paraíso, D. Córdova-Córdova 114 (UJAT).

Cedrela odorata L. Municipio: Balancán, D. Córdova-Córdova et al. 08 (UJAT); M. Juárez 245 (UJAT). Municipio: Centro, M. A. Frías y A. M. De la Cruz 146 (UJAT); D. Córdova-Córdova et al. 02 (UJAT). Municipio: Comalcalco, G. López 21 (UJAT). Municipio: Jalapa, S. Hernández y A. EspejoSerna 132 (UJAT). Municipio: Macuspana, M. I. Cruz 33 (UJAT). Municipio: Nacajuca, R. Ortega y W. Márquez 933 (UJAT); P. Gómez 1 (UJAT); D. Córdova-Córdova y C. M. Burelo-Ramos 36 (UJAT). Municipio: Teapa, S. Zamudio-Ruiz 375 (UJAT).

Guarea glabra Vahl. Municipio: Balancán, A. Ramírez 227 (UJAT). Municipio: Centro, J. M. Ascencio-Rivera 100 (UJAT); J. M. Ascencio-Rivera et al. 127 (UJAT); O. Castillo-Acosta 107 (UJAT); M. Á. Guadarrama-Olivera y M. A. Magaña-Alejandro 637 (UJAT); M. A. Magaña-Alejandro 61 (UJAT); M. A. Magaña-Alejandro 1721 (UJAT); A. Sol-Sánchez y J. M. Ascencio-Rivera 90 (UJAT). Municipio: Centla, COVINCE 7 (UJAT). Municipio: Huimanguillo, A. Ramón 23 (UJAT). Municipio: Jonuta, G. Ortiz-Gíl s.n. (UJAT). Municipio: Macuspana, L. Ruiz et al. 17 (UJAT); O. Castillo-Acosta 763 (UJAT); G. Ortiz-Gíl et al. 7097 (UJAT). Municipio: Tacotalpa, M. Hernández 154 (UJAT); M. Hernández 359 (UJAT); G. Ramos et al. 489 (UJAT); N. García y S. Ramírez 12 (UJAT). Municipio: Teapa, V. Pérez y N. Muñiz-Chavarría 14, (UJAT); V. Pérez y N. Muñiz-Chavarría 87 (UJAT); G. Ramos 693 (UJAT); A. M. Hanan-Alipi et al. 340 (UJAT); A. M. Hanan-Alipí y A. Sol-Sánchez 426 (UJAT); A. M. Hanan-Alipí 684 (UJAT); G. Ruíz y grupo de Fanerógamas 31 (UJAT). Municipio: Tenosique, N. C. Jiménez-Pérez y E. Pérez 580 (UJAT); N. C. Jiménez-Pérez y E. Pérez 587 (UJAT).

Guarea grandifolia DC. Municipio: Centro, J. M. Ascencio-Rivera y A. Sol-Sánchez 63 (UJAT); D. Córdova-Córdova y C. M. Burelo-Ramos 06 (UJAT); D. Córdova-Córdova y C. M. Burelo-Ramos 07 (UJAT); D. Córdova-Córdova y C. M. Burelo-Ramos 19 (UJAT); S. Zamudio-Ruiz 1401 (UJAT). Municipio: Teapa, A. M. Hanan-Alipi 491 (UJAT); A. M. Hanan-Alipi 583 (UJAT); G. Ortiz-Gíl y N. C. Jiménez-Pérez s.n. (UJAT); V. Pérez y N. MuñizChavarría 86 (UJAT); V. Pérez y N. Muñiz-Chavarría 88 (UJAT); V. Pérez y N. Muñiz-Chavarría 117 (UJAT); V. Pérez y N. Muñiz-Chavarría 154 (UJAT); V. Ramos-Jiménez y A. Sol-Sánchez 283 (UJAT); G. Ramos 751 (UJAT). Municipio:Tenosique, N. C. Jiménez-Pérez et al. 2000 (UJAT).

Melia azedarach L. Municipio: Centla, M. Á. Guadarrama-Olivera y E. López 886 (UJAT, MEXU). Municipio: Comalcalco, M. A. Magaña-Alejandro y Suárez 636 (CSAT, MEXU). Municipio: Cunduacán, Ventura 20067 (UNAM). Municipio: Huimanguillo, Ricardez 279 (MEXU). Municipio: Jalapa, C. Cowan 1990 (CSAT, MEXU). Municipio: Nacajuca, Calzada y C. Cowan 4952 (MEXU, XAL).

Swietenia macrophylla King. Municipio: Balancán, D. Córdova-Córdova et al. 11 (UJAT); G. Ortiz-Gíl 16 (UJAT). Municipio: Cárdenas, R. Canul 2 (UJAT). Municipio: Centro, D. Córdova-Córdova et al. 27 (UJAT); A. de la Cruz 03 (UJAT); M. A. Frías y N. Muñiz-Chavarría 27 (UJAT). Municipio: Teapa, V. Pérez y N. Muñiz-Chavarría 73 (UJAT).

*Trichilia breviflora Blake \& Standl. Municipio: Teapa, M. Á. Guadarrama-Olivera y G. Ortiz-Gíl 95 (UJAT).

Trichilia havanensis Jacq. Municipio: Balancán, M. Juárez 263 (UJAT); A. Ramírez 111 (UJAT); A. Ramírez 256 (UJAT). Municipio: Cárdenas, G. Ortiz-Gíl 2087 (UJAT). Municipio: Centro, O. Castillo-Acosta y J. M. Ascencio-Rivera 132 (UJAT); D. Córdova-Córdova 28 (UJAT); D. CórdovaCórdova y C. M. Burelo-Ramos 13 (UJAT); D. Córdova-Córdova y C. M. Burelo-Ramos 14 (UJAT); M. A. Magaña-Alejandro 1401 (UJAT); A. Sol-Sánchez y J. M. Ascencio-Rivera 96 (UJAT). Municipio: Comalcalco, D. Córdova-Córdova et al. 38 (UJAT). Municipio: Cunduacán, A. NoveloRetana 1684 (UJAT, MEXU). Municipio: Jalpa de Méndez, D. Córdova-Córdova et al. 37 (UJAT). Municipio: Paraíso, D. Córdova-Córdova et al. 40 (UJAT). Municipio: Tacotalpa, P. Díaz-Jiménez y A. Lastra 64 (UJAT); D. Carrillo 35 (UJAT). D. Córdova-Córdova y C. M. Burelo-Ramos 077 (UJAT). Municipio: Teapa, J. Calónico 21201 (UJAT); D. Córdova-Córdova et al. 31 (UJAT); M. A. Magaña-Alejandro y S. Zamudio-Ruiz 1015 (UJAT); V. Ramos-Jiménez 197 (UJAT). Municipio: Tenosique, O. Castillo-Acosta 620 (UJAT); O. Castillo-Acosta 622 (UJAT); N. C. Jiménez-Pérez 1679 (UJAT).

Trichilia hirta L. Municipio: Balancán, D. Córdova-Córdova et al. 12 (UJAT); D. Córdova-Córdova et al. 33 (UJAT). Municipio: Centro, V. RamosJiménez 116 (UJAT). Municipio: Cunduacán, A. Novelo-Retana 1690 (UJAT). Municipio: Emiliano Zapata, D. Córdova-Córdova et al. 35 (UJAT). Municipio: Teapa, E. Ventura 21222 (UJAT). Municipio: Tenosique, C. M. Burelo-Ramos y M. Campos-Díaz 631 (UJAT); M. T. Germán 21222 (MEXU); N. C. Jiménez-Pérez 1681 (UJAT). 
Apéndice: Continuación.

Trichilia martiana C. DC. Municipio: Centro, J. M. Ascencio-Rivera et al. 112. (UJAT); D. Córdova-Córdova y C. M. Burelo-Ramos 16 (UJAT). Municipio: Comalcalco, D. Córdova-Córdova y C. M. Burelo-Ramos 39 (UJAT). Macuspana, M. Á. Guadarrama-Olivera y M. A. Magaña-Alejandro 597 (UJAT). Municipio: Nacajuca, J. I. Calzada. 4963 (UJAT). Municipio: Tacotalpa, M. Á. Guadarrama-Olivera y C. M. Burelo-Ramos 6984 (UJAT); A. Lastra. O y P. Díaz-Jiménez 121 (UJAT); G. Ramos 701 (UJAT). Municipio: Teapa, D. Córdova-Córdova et al. 32 (UJAT); M. Á. GuadarramaOlivera et al 944 (UJAT); M. Á. Guadarrama-Olivera. 1339 (UJAT); M. Á. Guadarrama-Olivera 95.9.175 (UJAT); M. Á. Guadarrama-Olivera y G. Ortiz-Gíl 95.5.88 (UJAT); A. M. Hanan-Alipi 534 (UJAT); V. Pérez y N. Muñiz-Chavarría 38 (UJAT); V. Pérez y N. Muñiz-Chavarría 45 (UJAT); V. Pérez y N. Muñiz-Chavarría 65 (UJAT); V. Pérez y N. Muñiz-Chavarría 92 (UJAT); V. Ramos-Jiménez 407 (UJAT); G. Ramos y M. Á. GuadarramaOlivera 675 (UJAT); V. Ramos-Jiménez 407 (UJAT); F. Ventura A. 20655 (UJAT); F. Ventura A. 21185 (UJAT).

Trichilia moschata Sw. Municipio: Jalpa de Méndez, M. A. Magaña-Alejandro s.n. (UJAT).

Trichilia pallida Sw. Municipio: Huimanguillo, J. G. García y E. J. Palma 214 (UJAT).

*Trichilia trifolia L. Municipio: Balancán, J. G. García y E. J. Palma 117 (MEXU). 